\title{
A GRAPHICAL METHOD FOR GREAT CIRCLE ROUTES
}

\author{
Tien-Pen Hsu' ${ }^{1)}$, Ph.D. \\ Chih-Li Chen ${ }^{2)}$, Ph.D. \\ Tsung-Hsuan Hsieh ${ }^{1)}$, Ph.D. \\ ${ }^{1)}$ Department of Civil Engineering, National Taiwan University, Taiwan \\ ${ }^{2)}$ Merchant Marine Department, National Taiwan Ocean University, Taiwan
}

\begin{abstract}
A great circle route (GCR) is the shortest route on a spherical earth model. Do we have a visual diagram to handle the shortest route? In this paper, a graphical method (GM) is proposed to solve the GCR problems based on the celestial meridian diagram (CMD) in celestial navigation. Unlike developed algebraic methods, the GM is a geometric method. Appling computer software to graph, the GM does not use any equations but is as accurate as using algebraic methods. In addition, the GM, which emphasizes the rotational surface, can depict a GCR and judge its benefit.
\end{abstract}

Keywords: transoceanic shipping; great circle route; waypoints; celestial meridian diagram

\section{INTRODUCTION}

The shortest route is an important topic of maritime transportation, especially in transoceanic shipping. In recent years, the trend of maritime transportation shows that: the fluctuations in energy price may influence the shipping strategies and developing projects. For example, when oil price began to rise in 2009, slow steaming (a major cost-cutting measure) was used to adapt the high price, and energy-efficient ships were developed in vogue. When the price of oil dropped in June 2014, ships resumed sailing at faster speeds to increase their cargo capacity, and the use of energy-efficient ships was decreased [14]. However, whether the price of oil are rising or dropping, shipping companies can get benefit by sailing on the shortest route.

The great circle route (GCR) is the shortest route on a spherical earth model, and the great ellipse route (GER) is the shortest route on a spheroidal earth model. The WGS 84 spheroidal model is commonly be used in modern navigation system, and the spherical model is commonly be used for instruction and simplified calculation. Hence, the great circle route (GCR) has still been discussing continuously. Most navigation textbooks have used Napier's rules of the rightangled spherical triangle to deal with GCR problems $[1,7,11]$. To reduce calculation steps and to apply to different situations, many researches have been published. For example, Miller et al. (1991), Nastro and Tancredi (2010), and Tseng and Chang (2014) used linear combination to obtain equations $[9,10$, 13]. Chen et al. (2004), Earle (2005), Chen et al. (2014) and Chen (2016) used vector algebra to yield equations $[2,4,5,8]$. Chen et al. (2015) used rotation transformation to produce equations [3]. Actually, these studies, which creatively use different approaches to derive equations, are all algebraic methods.

Unlike these developed algebraic methods, we propose a geometric method, namely graphical method (GM), to solve GCR problems. In celestial navigation, the celestial meridian diagram (CMD) is an important tool to analyze the position of a heavenly body on the celestial sphere. However, angle 
deviations on the diagram by hand-made may reach 2 degrees [12]. To overcome the disadvantage, we use computer software to draw the diagram, which obtained results are as accurate as using algebraic equations. Hence, we can use the GM to solve GCR problems without using any equations.

Comparing with the algebraic methods, the GM has two advantages. The one advantage is that it has an outstanding ability to display the GCR. The other advantage is that it can analyze the geographic relationship graphically. Thus, The GM not only can demonstrates a GCR on the diagram more direct and clear, but also can help us to analyze the relative variables and judge the benefit of GCR.

\section{THEORETICAL BACKGROUNDS}

Based on the concepts of CMD, we propose the GM to obtain the relative information of GCR. The CMD provides two important concepts as follows.

The first concept is the technique which fixed position by combining two coordinate systems. In celestial navigation, the position of any heavenly body $(S)$ can be presented in the celestial equator system or in the celestial horizon system. As shown in Fig. 1, the red symbols and lines represent the celestial equator system, and the blue symbols and lines represent the celestial horizon system.

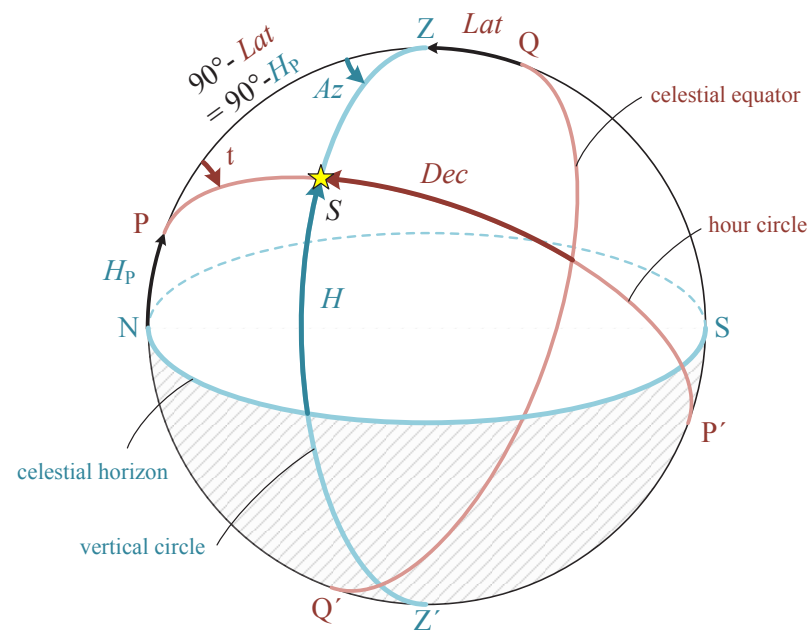

Fig. 1. Celestial coordinate systems

From the viewpoint of the north celestial pole $(P)$, the position of $S$ is fixed by using declination $(D e c)$ and meridian angle $(t)$ in the celestial equator system. From the viewpoint of the observer's zenith $(Z)$, the position of $S$ is fixed by using altitude $(H)$ and azimuth angle $(A z)$ in the celestial horizon system. Because the observer's latitude (Lat) is equal to the altitude of the celestial pole $(H \mathrm{p})$, the CMD cleverly combines two coordinate systems. Therefore, when a coordinate in one system is known, its position in another system can be determined on the CMD.
Applying this concept to the GCR, when the earth coordinate system is known, we can construct a horizon coordinate system and use variables of GCR to replace variables of heavenly body. For instance, North Pole $\left(\mathrm{P}_{\mathrm{N}}\right)$ replaces north celestial pole $(\mathrm{P})$, departure $(\mathrm{F})$ replaces observer's zenith $(\mathrm{Z})$, and destination $(T)$ replace heavenly body $(S)$.

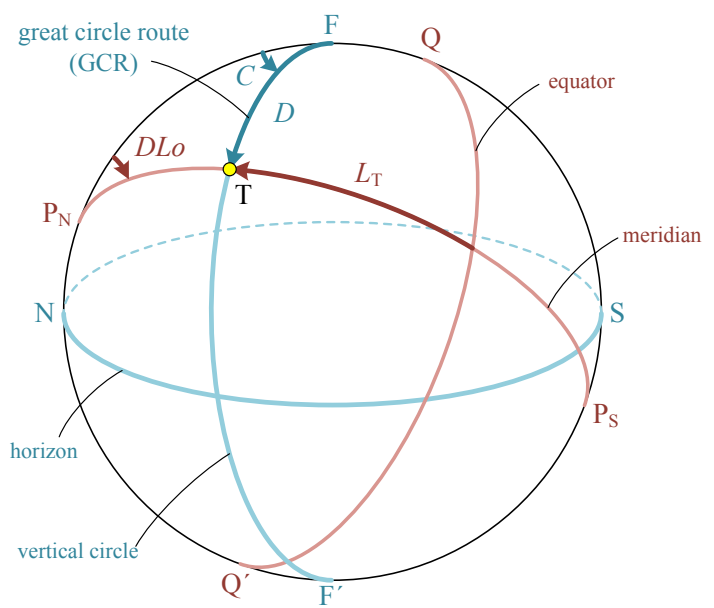

Fig. 2. Combinded coordinate systems of the GCR

As shown in Fig. 2, the red symbols and lines represent the earth coordinate system, and the blue symbols and lines represent the horizon coordinate system. From the viewpoint of the North Pole $\left(\mathrm{P}_{\mathrm{N}}\right)$, the position of destination $(\mathrm{T})$ is fixed by using latitude $\left(L_{\mathrm{T}}\right)$ and difference of longitude $(D L o)$ in the earth coordinate system.
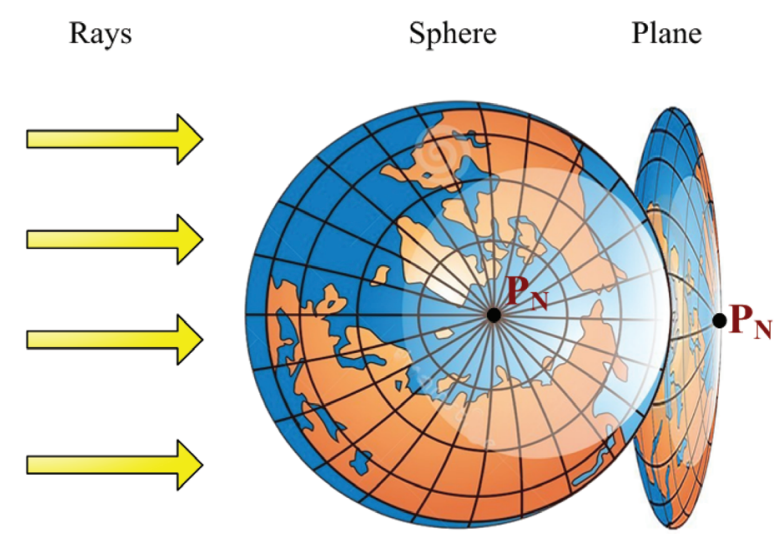

Fig. 3. An illustration of the azimuthal orthographic projection (equatorial aspect)

From the viewpoint of the departure (F), the position of destination $(\mathrm{T})$ is fixed by using great circle distance $(D)$ and initial course angle $(C)$ in the horizon coordinate system. Hence, when the destination's latitude and longitude in the earth coordinate system are known, great circle distance and initial course angle can be obtained in the horizon coordinate system. 
a) Sphere

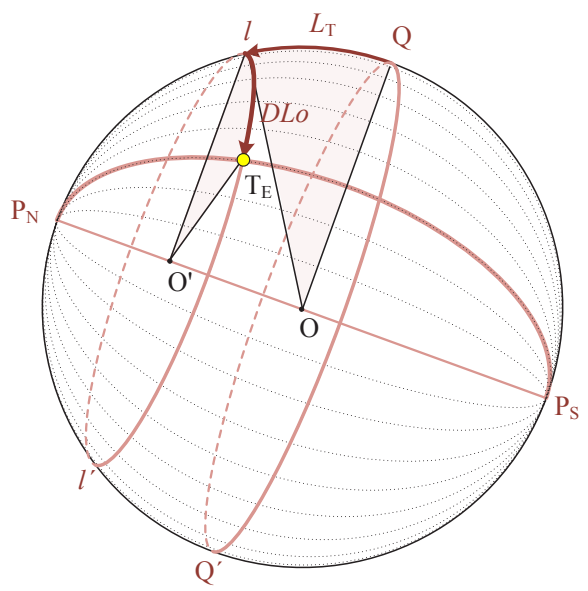

b) Plane

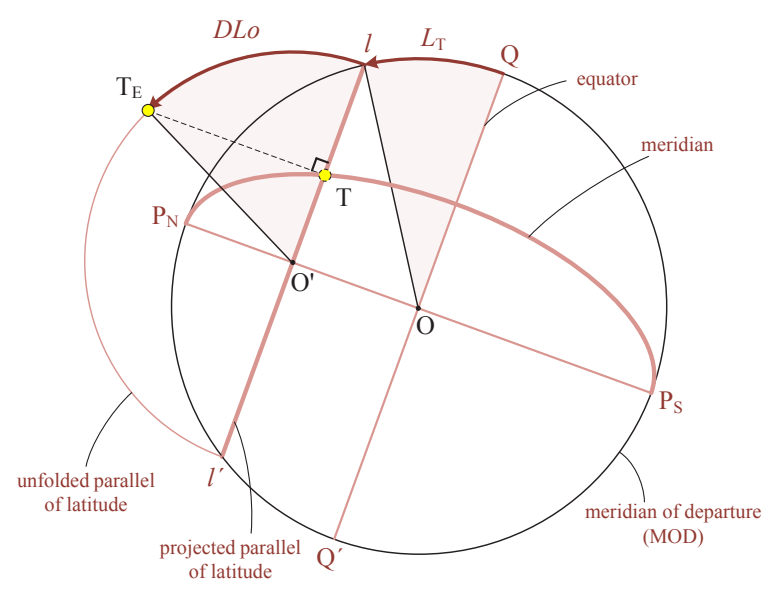

Fig. 4. Projecting the earth coordinate system on a plane

The second concept is the technique of azimuthal orthographic projection (equatorial aspect), which makes the arcs and angles on the sphere can be measured on the plane. As shown In Fig. 3, the hemisphere of the earth is irradiated by the light rays at infinite distance from the earth. The equator is in the centerline, and the sphere is projected onto a plane. The projection looks like that someone took a picture of the earth from space. The projected earth coordinate system is shown in Fig. 4, and the projected horizon coordinate system is shown in Fig. 5. In Fig. 4(b), the projected meridian of departure (MOD) would appear as the outer limit circle. According to the angle values which measure from the outer limit circle to the projected meridians, the meridian whose angle is $90^{\circ}$ would appear as a diameter $\left(\overline{\mathrm{P}_{\mathrm{N}} \mathrm{P}_{\mathrm{S}}}\right)$, the others would appear as curved lines (e.g., $\widehat{\mathrm{P}_{\mathrm{N}} \mathrm{P}_{\mathrm{S}}}$ ). The projected equator would appear as a diameter $\left(\overline{\mathrm{QQ}^{\prime}}\right)$. The all projected parallels of latitude (POLs) would appear as straight lines (e.g., $l l^{\prime}$ ), which are parallel to the equator. They also can unfold as semicircles on the projection plane (e.g., $l l$ ) ). In Fig. 5(b), the projected principal vertical of the departure would appear as the outer limit circle (this circle also represented as the projection of the MOD in the earth coordinate system). According to the angle values which measure from the outer limit circle to the projected vertical circles, the vertical circle whose angle is $90^{\circ}$ would appear as a diameter $\left(\overline{\mathrm{FF}^{\prime}}\right)$ (this vertical circle also called the prime vertical), the others would appear as curved lines (e.g., FF'). The GCR is the part of the vertical circle, which is from the departure to the destination (e.g., $\widehat{\mathrm{FT}})$. The projected horizon would appear as a diameter $(\overline{\mathrm{NS}})$ which includes all azimuths (the north is at the left, the south is at the right, the west is at the center, and the east is at the "back" side of the center). The all projected distance circles (each point on the same circle has the same distance from the departure to itself) would appear as straight lines (e.g., $\overline{d d^{\prime}}$ ). They also can unfold as semicircles on the projection plane (e.g., $\left.d d^{\prime}\right)$. Furthermore, when the great circle plane or the small circle plane on the sphere is orthogonal or parallel to the light rays, their arcs and angles can be measured on the projection plane. Therefore, in Fig. 4(b), a latitude (e.g., $L_{\mathrm{T}}$ ) can be found by measuring the central angle of the projected MOD (e.g., $\angle Q O l$ ). A difference of longitude (e.g., $D L o$ ) can be obtained by measuring the central angle of the spherical POL (e.g., $\angle l O^{\prime} \mathrm{T}_{\mathrm{E}}$ ). In Fig. 5(b), a great circle distance (e.g., $D$ ) can be found by measuring the central angle of the projected principal vertical (e.g., FOd ). A great circle initial course (e.g., C) can be obtained by measuring the central angle of the spherical distance circle (e.g., $\angle d \mathrm{O}^{\prime} \mathrm{T}_{\mathrm{H}}$ ).

a) Sphere

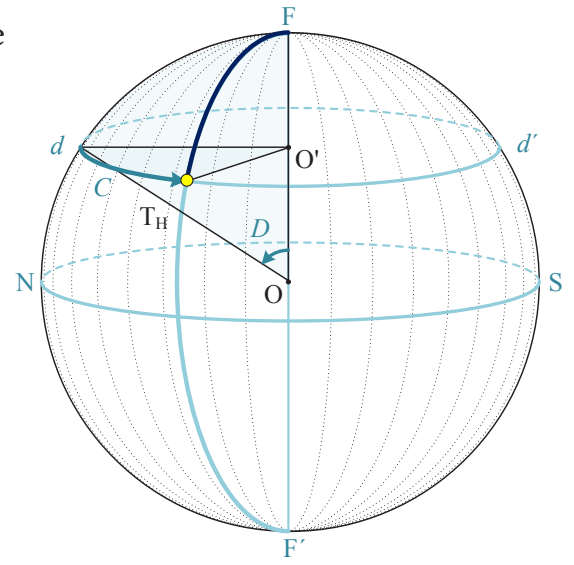

b) Plane

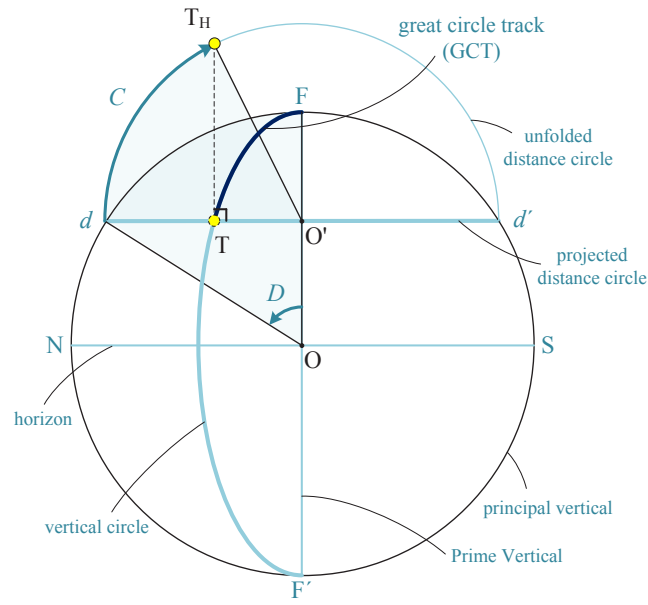

Fig. 5. Projecting the horizon coordinate system on a plane 


\section{GRAPHICAL METHOD}

The latitude of departure $\left(L_{\mathrm{F}}\right)$, the latitude of destination $\left(L_{\mathrm{T}}\right)$, and the difference of longitude between departure and destination ( $D L o$ ) are known. How the GM solved the GCR problems are illustrated as follows:

\section{FUNDAMENTAL DIAGRAM}

\section{Step 1: construct the fixed horizon coordinate system}

As shown in Fig. 6, graph a circle whose center is $\mathrm{O}$ and radius is $r$. Locate four points ( $F, F^{\prime}, N$ and $S$ ) on the circle's up, down, left, and right direction position. Draw the projected prime vertical $\left(\overline{\mathrm{FF}^{\prime}}\right)$ which goes through the point $\mathrm{F}$ and $\mathrm{F}^{\prime}$, and draw the projected horizon $(\overline{\mathrm{NS}})$ which goes through the point $\mathrm{N}$ and $\mathrm{S}$. Thus, the two axes of horizon coordinate are established.

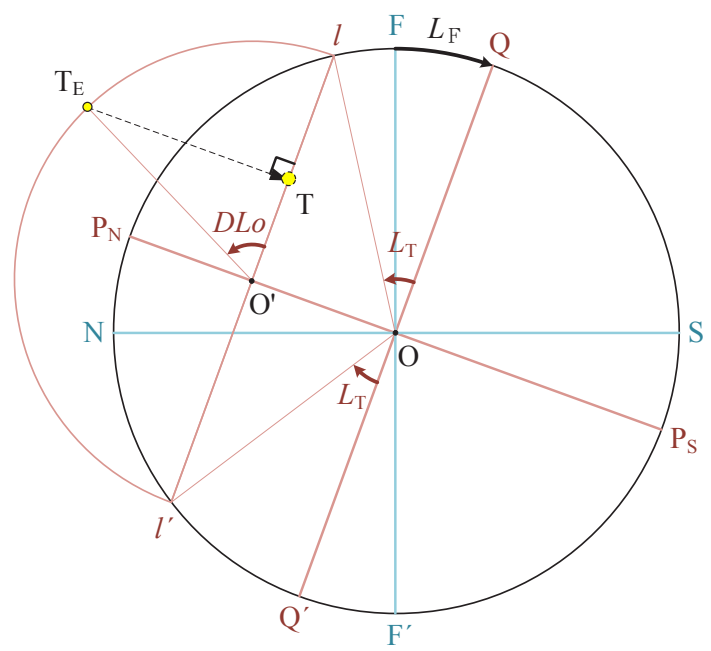

Fig. 6. Locating the destination in the earth coordinate

\section{Step 2: derive the earth coordinate system}

As shown in Fig. 6, the point Q can be derived from measuring an arc of $L_{\mathrm{F}}$ which starts from the point $\mathrm{F}$ along the MOD. Due to the point $\mathrm{Q}$ is on the equator, when the $L_{\mathrm{F}}$ is in the north latitude, the point $\mathrm{Q}$ will be on its south side and vice versa. For example, when the $L_{\mathrm{F}}$ is $20^{\circ} \mathrm{N}$, the point Q can be found by measuring an arc of $20^{\circ}$ which starts from the point $\mathrm{F}$ towards the point $S$. The point $\mathrm{Q}^{\prime}$ can be plotted by rotating the point $\mathrm{Q} 180^{\circ}$ around the center of $\mathrm{O}$. The projected equator ( $\left.\mathrm{QQ}^{\prime}\right)$ can be obtained by drawn a line which goes through the point Q and Q'. Then, draw the projected polar axis $\left(\overline{\mathrm{P}_{\mathrm{N}} \mathrm{P}_{\mathrm{S}}}\right)$ which is perpendicular to the equator. The north pole $\left(\mathrm{P}_{\mathrm{N}}\right)$ is the endpoint which lies closest to the north, and the south pole $\left(\mathrm{P}_{\mathrm{S}}\right)$ is the other endpoint which lies closest to the south. Thus, the two axes of earth coordinate are established.

\section{Step 3: locate the destination position in the earth coordinate system}

As shown in Fig. 6, draw the point $l$ from measuring an $\operatorname{arc}$ of $L_{\mathrm{T}}$ which starts from the point $\mathrm{Q}$ along the MOD, and draw the point $l^{\prime}$ from measuring an $\operatorname{arc}$ of $L_{\mathrm{T}}$ which starts from the point Q' along the MOD. The projected POL ( $\left.l l^{\prime}\right)$ can be obtained by drawn a line which goes through the point $l$ and $l^{\prime}$. Due to the point $l$ and $l^{\prime}$ are on the POL, when the $L_{\mathrm{T}}$ is in the north latitude, the point $l$ and $l^{\prime}$ will be on the equator's north side and vice versa. For example, when the $L_{\mathrm{T}}$ is $32.5^{\circ} \mathrm{N}$, the point $l$ can be found by measuring an arc of $32.5^{\circ}$ which starts from the point $\mathrm{Q}$ towards the point $\mathrm{N}$. Then, draw the spherical POL $\left(l l^{\prime}\right)$ by graphing a small circle whose center is $\mathrm{O}^{\prime}$ (the intersection of $l l^{\prime}$ and $\overline{\mathrm{P}_{\mathrm{N}} \mathrm{P}_{\mathrm{S}}}$ ) and radius is $\overline{O^{\prime} l}$. The point $\mathrm{T}_{\mathrm{E}}$ can be derived from measuring an arc of $D L o$ which starts from the point $l$ along the small circle. Due to the point $\mathrm{T}_{\mathrm{E}}$ only decided by the angle of $D L o$, no matter the DLo is in the west longitude or in the east longitude, the position of point $T_{E}$ which obtained on the small circle is the same. Then, the point $\mathrm{T}_{\mathrm{E}}$ can be projected back onto the tangent plane to mark the point T. Therefore, the destination's position is located on the diagram.

\section{GCR PROBLEMS}

1) Find the great circle distance and the initial course

When the destination $(\mathrm{T})$ is located, the great circle distance and the initial course can be measured in the horizon coordinate system. As shown in Fig. 7, draw the projected distance circle of the destination $\left(d d^{\prime}\right)$, which goes through the point $\mathrm{T}$ and is parallel to the horizon (NS). The point $d$ is the endpoint which lies closest to the north, and the point $d^{\prime}$ is the other endpoint which lies closest to the south. The great circle distance $(D)$ can be obtained by measuring an arc of $\angle \mathrm{FO} d$ (or $\angle \mathrm{FO} d^{\prime}$ ) which starts from the departure towards the point $d$ (or the point $d$ ) along the MOD. The measured degrees should convert into minutes because the unit of the great circle distance is the nautical miles (nm). For example, when the $\angle \mathrm{FO} d$ is $57.3^{\circ}$, the great circle distance is $3438 \mathrm{~nm}$.

Besides, draw the spherical distance circle of the destination by graphing a small circle whose center is $\mathrm{O}^{\prime}$ (the intersection of $d d^{\prime}$ and $\overline{\mathrm{FF}}$ ) and radius is $\overline{\mathrm{O}^{\prime} d}$. The point $\mathrm{T}_{\mathrm{H}}$ can be plotted by returning the point $\mathrm{T}$ on the small circle. The great circle initial course angle can be obtained by measuring an arc of $\angle d \mathrm{O}^{\prime} \mathrm{T}_{\mathrm{H}}$ (or $\angle d^{\prime} \mathrm{O}^{\prime} \mathrm{T}_{\mathrm{H}}$ ) value which starts from the point $d$ (or the point $d^{\prime}$ ) towards the point $\mathrm{T}_{\mathrm{H}}$ along the $d d$ (use the $\angle d \mathrm{O}^{\prime} \mathrm{T}_{\mathrm{H}}$ when the $L_{\mathrm{F}}$ is in the north latitude and use the $\angle d^{\prime} \mathrm{O}^{\prime} \mathrm{T}_{\mathrm{H}}$ when the $L_{\mathrm{F}}$ is in the south latitude). Due to the prefix of initial course angle is consistent with the latitude of departure $\left(L_{\mathrm{F}}\right)$ and the suffix of initial course angle is consistent with the difference of longitude $(D L o)$, the initial course angle $(C)$ can convert into the initial course $\left(C_{n}\right)$. For example, when the $\angle d \mathrm{O}^{\prime} \mathrm{T}_{\mathrm{H}}$ is $63.5^{\circ}$, the $C$ is $\mathrm{N} 63.5^{\circ} \mathrm{W}$, and the great circle initial course $\left(C_{n}\right)$ is $296.5^{\circ}$. 


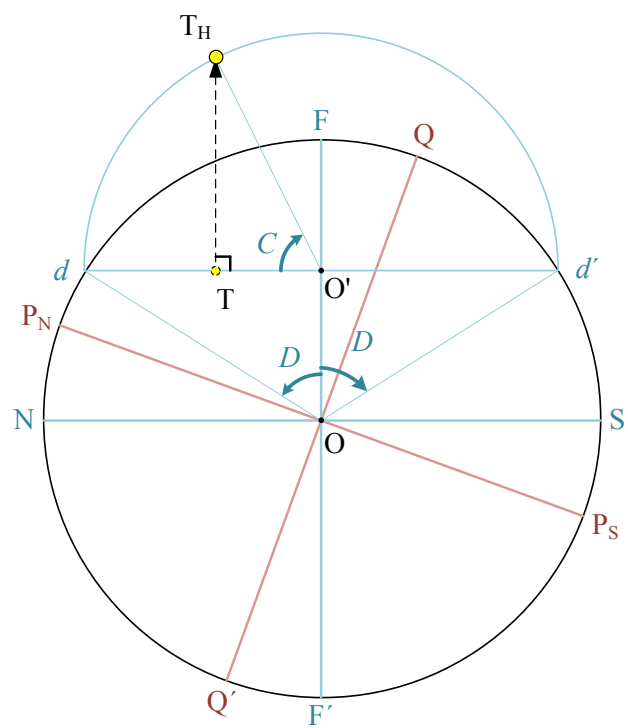

Fig. 7. Measuring the destination in the horizon coordinate

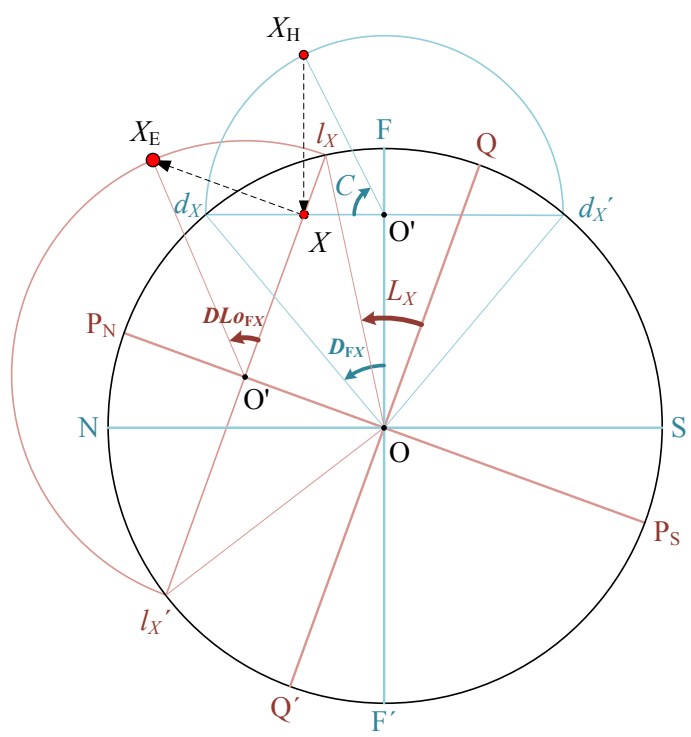

Fig. 8. Finding the waypoint under condition 1

\section{2) Find waypoints}

- Condition 1: giving the great circle distance to obtain the waypoint position

When the great circle distance, which starts from the departure towards the waypoint $\left(D_{\mathrm{FX}}\right)$, and the initial course angle $(C)$ are given, the waypoint can be located in the horizon system. As shown in Fig. 8, draw the projected distance circle of the waypoint $\left(\overline{d_{X} d_{X}{ }^{\prime}}\right)$, which is parallel to the horizon (NS). The point $d_{X}$ can be found by measuring an $\operatorname{arc}$ of $D_{F X}$ which starts from the point $\mathrm{F}$ towards the point $\mathrm{N}$, and the point $d_{X}{ }^{\prime}$ can be found by measuring an arc of $D_{F X}$ which starts from the point $\mathrm{F}$ towards the point $\mathrm{S}$. Besides, draw the spherical distance circle of the waypoint $\left(\widehat{d_{X} d_{X}}\right)$ by graphing a small circle whose center is $\mathrm{O}^{\prime}$ (the intersection of $\overline{d_{X} d_{X}{ }^{\prime}}$ and $\overline{\mathrm{FF}^{\prime}}$ ) and radius is $\overline{\mathrm{O}^{\prime} d_{X}}$. The point $X_{\mathrm{H}}$ can be marked by measuring an arc of $\mathrm{C}$ which starts from the point $d_{X}$ along the small circle $\left(\widehat{d_{X} d_{X}}\right)$. The point $X$ can be marked by projecting the point $X_{\mathrm{H}}$ back onto the tangent plane.

When the waypoint $(X)$ is located, its latitude and longitude can be measured in the earth coordinate system. Draw the projected POL of the waypoint $\left(\overline{l_{X} l_{X}{ }^{\prime}}\right)$ which goes through point $X$ and is parallel to the equator ( $\left.\overline{\mathrm{QQ}^{\prime}}\right)$. The latitude of the waypoint can be obtained by measuring an arc of $\angle \mathrm{QO} l_{X}$ (or $\angle Q^{\prime} \mathrm{O} l_{X}{ }^{\prime}$ ) along the MOD. Besides, draw the spherical POL of the waypoint $\left(\widehat{l}_{X} l_{X}^{\prime}\right)$ by graphing a small circle whose center is $\mathrm{O}^{\prime}$ (the intersection of $\overline{l_{X} l_{X}{ }^{\prime}}$ and $\overline{\mathrm{P}_{\mathrm{N}} \mathrm{P}_{S}}$ ) and radius is $\overline{\mathrm{O}^{\prime} l_{X}}$. The point $X_{\mathrm{E}}$ can be marked by returning the point $\mathrm{X}$ on the small circle $\left(l_{X} l_{X}{ }^{\prime}\right)$. Therefore, the difference of longitude between the departure and the waypoint $\left(D L o_{\mathrm{FX}}\right)$ can be obtained by measuring an arc of $\angle Q^{\prime} \mathrm{O} l_{X}$ along the small circle. For example, when the $\angle \mathrm{QO} l_{X}$ is $32.1^{\circ}$, the latitude of the waypoint $\left(L_{X}\right)$ is $32^{\circ} 06.0^{\prime} \mathrm{N}$. When the $\angle l_{X} O^{\prime} X_{E}$ is $42.8^{\circ}$, the difference of longitude between the departure and the waypoint $\left(D L o_{\mathrm{FX}}\right)$ is $42^{\circ} 48.0^{\prime} \mathrm{W}$.

- Condition 2: giving the longitude of the waypoint to obtain its latitude

When the great circle initial course angle $(C)$ and the difference of longitude between departure and waypoint $\left(D L o_{\mathrm{FX}}\right)$ are given, the waypoint can be located on the intersection of its vertical circle ( $\widehat{\mathrm{FF}}$, the blue curved line) and meridian $\left(\widehat{\mathrm{P}_{\mathrm{N}} \mathrm{P}_{\mathrm{S}}}\right.$, the red curved line) as shown in Fig. 9. At first, sketch out the vertical circle of the waypoint. Assume that there are three points on the vertical circle, and their great circle distance are respectively $1800 \mathrm{~nm}\left(30^{\circ}\right), 3600 \mathrm{~nm}\left(60^{\circ}\right)$ and $5400 \mathrm{~nm}\left(90^{\circ}\right)$. The three spherical distance circles can be drawn. The points $G_{1 \mathrm{H}}, G_{2 \mathrm{H}}$, and $G_{3 \mathrm{H}}$ can be obtained by respectively measuring an arc of $C$ along these small circles. The points $G_{1}, G_{2}$, and $G_{3}$ can be marked by projecting the point $G_{1 \mathrm{H}}, G_{2 \mathrm{H}}$, and $G_{3 \mathrm{H}}$ back onto the tangent plane. Hence, the vertical circle of the waypoint can be sketched out by linking the points $G_{1}, G_{2}$, and $G_{3}$. The more points be assumed, the more complete the vertical circle of the waypoint can be sketched out. Next, sketch out the meridian of the waypoint. Assume that there are three points on the meridian, and their latitudes are respectively $60^{\circ} \mathrm{N}, 30^{\circ} \mathrm{N}$ and $0^{\circ} \mathrm{N}$. The three spherical POLs can be drawn. The three points can similarly be marked by respectively measuring an arc of $D L o_{F X}$ along these small circles. The points $M_{1} M_{2}$, and $M_{3}$ can be marked by projecting these marked points back onto the tangent plane. Hence, the meridian of the waypoint can be sketched out by linking the points $M_{1} M_{2}$, and $M_{3}$. Accordingly, the intersection of these two sketched out curved lines is the waypoint position. The latitude of the waypoint $\left(L_{X}\right)$ can obtained by measuring an arc of $\angle Q O l_{X}$ (or $\angle Q^{\prime} \mathrm{O}_{X}{ }_{X}{ }^{\prime}$ ) along the MOD. For example, when the $C$ is $N 63.5^{\circ} \mathrm{W}$ and the $D L o_{F X}$ is $46^{\circ}$, the latitude of the waypoint $\left(L_{X}\right)$ is $32^{\circ} 24.0^{\prime} \mathrm{N}$.

- Condition 3: giving the latitude of the waypoint to obtain its longitude

When the latitude of the waypoint $\left(L_{X}\right)$ is given, the waypoint can be located on the intersection of its vertical 
circle $\left(\widehat{\mathrm{FF}}\right.$ the blue curved line) and POL $\left(\overline{\bar{l}_{X} l_{X}{ }^{\prime}}\right)$, the red straight line) as shown in Fig. 10. The vertical circle of the waypoint can be sketched out by assuming some different points which have different great circle distances just as in the Condition 2. The projected POL of the waypoint can be drawn by using the known latitude of the waypoint $\left(L_{X}\right)$. Accordingly, the intersection of the vertical circle and the POL is the waypoint position (the obtained point number is one or two). The point $X_{\mathrm{E}}$ can be marked by returning the chosen waypoint $(X)$ on the spherical POL of the waypoint $\left(\bar{l}_{X} \bar{l}_{X}^{\prime}\right)$. Therefore, the difference of longitude between the departure and the waypoint $\left(D L o_{\mathrm{FX}}\right)$ can be obtained by measuring an arc of $\angle l_{X} \mathrm{O}^{\prime} X_{E}$ along the small circle. For example, when the $C$ is $\mathrm{N} 63.5^{\circ} \mathrm{W}$ and the $L_{X}$ is $30^{\circ} \mathrm{N}$, the difference of longitude between the departure and the waypoint $\left(D L o_{\mathrm{FX}}\right)$ is $29^{\circ} 24.0^{\prime} \mathrm{W}$.

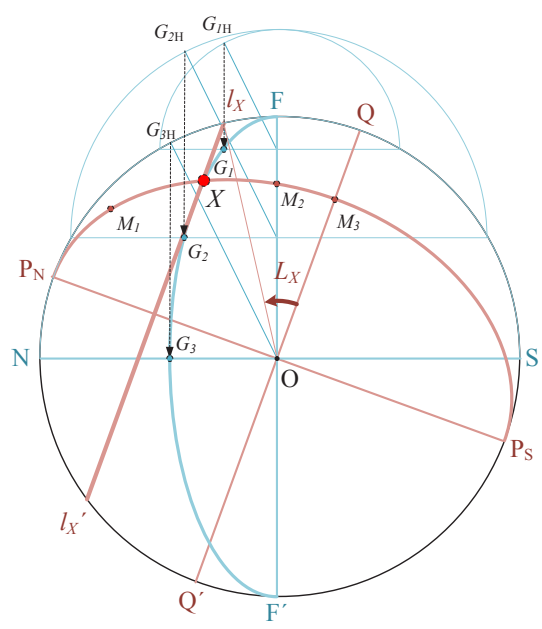

Fig. 9. Finding the waypoint under condition 2

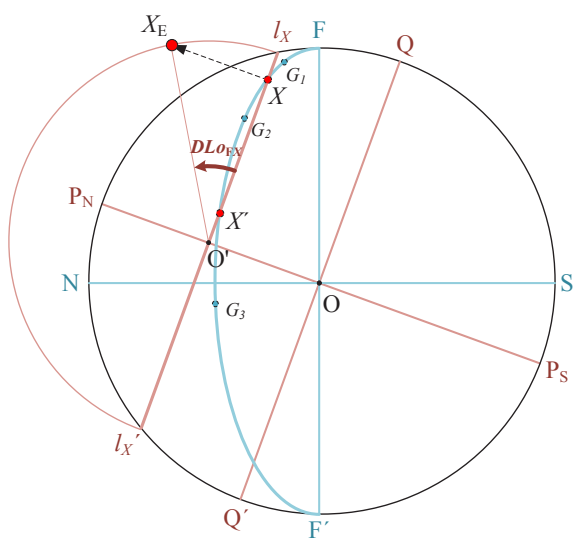

Fig. 10. Finding the waypoint under condition 3

3) Find the vertex and the equator crossing point

As shown in Fig. 11, the vertex is the point which has the highest latitude on a GCR. Draw the projected POL of the vertex $\left(\overline{l_{v} l_{v}^{\prime}}\right)$ which is tangent to the GCR and is parallel to the equator $\left(\overline{\mathrm{QQ}^{\prime}}\right)$. The vertex is the intersection of the GCR and the projected POL $\left(\overline{l_{V} l_{v}^{\prime}}\right)$. Its latitude $\left(L_{V}\right)$ and difference of longitude $\left(D L o_{\mathrm{FX}}\right)$ can be obtained by respectively measuring the angle of $\angle \mathrm{QO} l_{V}$ and $\angle l_{V} \mathrm{O}^{\prime} V_{\mathrm{E}}$. Besides, the equator crossing point is the intersection of the GCR and the equator $\left(\overline{\mathrm{QQ}^{\prime}}\right)$.
Its latitude is $0^{\circ}$ and its difference of longitude ( $D L o_{\mathrm{FE}}$ ) can be obtained by measuring the angle of $\angle \mathrm{QO} E_{\mathrm{E}}$. For example, when the $\angle \mathrm{QO} l_{\mathrm{v}}$ is $32.7^{\circ}$, the latitude of the vertex $\left(L_{V}\right)$ is $32^{\circ} 42.0^{\prime} \mathrm{N}$. When the $\angle l_{V} \mathrm{O}^{\prime} V_{\mathrm{E}}$ is $55.5^{\circ}$, the difference of longitude between the departure and vertex is $\left(D L o_{\mathrm{FV}}\right)$ $55^{\circ} 30.0^{\prime} \mathrm{W}$. When the $\angle \mathrm{QO} E_{\mathrm{E}}$ is $145.5^{\circ}$, the difference of longitude between the departure and equator crossing point $\left(D L o_{\mathrm{FE}}\right)$ is $145^{\circ} 30.0^{\prime} \mathrm{W}$.

Generally, the GCR between the departure (F) and the destination $(\mathrm{T})$ has at most one vertex and one equator crossing point. However, as shown in Fig. 11, a complete great circle has two vertices $\left(V_{1}\right.$ and $\left.V_{2}\right)$ and two equator crossing points $\left(E_{1}\right.$ and $\left.E_{2}\right)$. Their values can be obtained by the method described above.

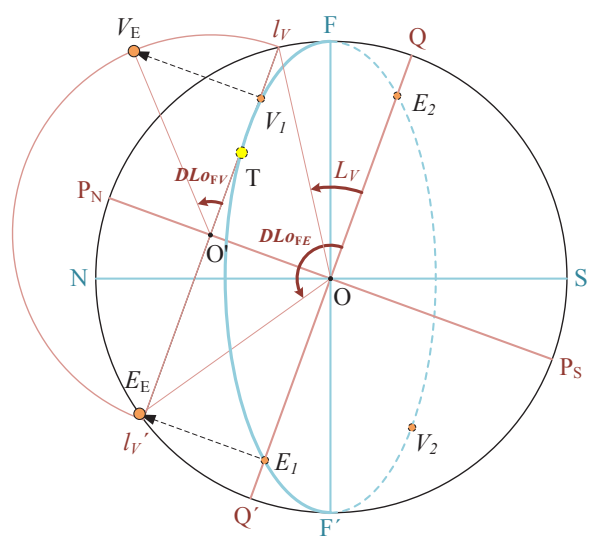

Fig. 11. Finding the vertex and the equator crossing point

\section{DEMONSTRATED EXAMPLE}

In this paper, the GeoGebra, which is a kind of computer graphing software, is used to execute the graphical method (GM) to solve GCR problems. Take a voyage as for instance, its interface is as shown in Fig. 12. A vessel is leaving from Sydney (AUSTRALIA) to Balboa (PANAMA). The latitude of departure $\left(L_{\mathrm{F}}\right)$ is $33^{\circ} 51.5^{\prime} \mathrm{S}$, the latitude of destination $\left(L_{\mathrm{T}}\right)$ is $08^{\circ} 53.0^{\prime} \mathrm{N}$, and the difference of longitude between departure and destination $(D L o)$ is $129^{\circ} 16.0^{\prime} \mathrm{E}$ [4]. Following questions are calculated by using the GeoGebra.

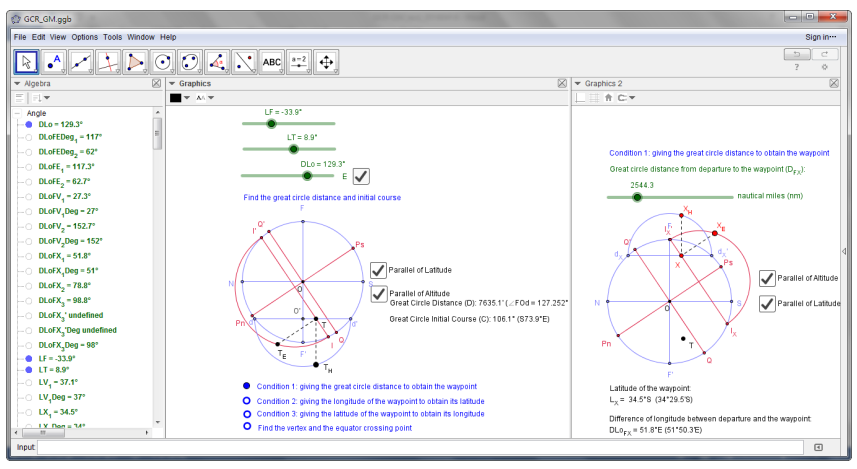

Fig. 12. Interface of the GeoGebra 
1) Find the great circle distance $(D)$ and the initial course $C_{n}$. As shown in Fig. 13, the results of calculation are that the great circle distance $(D)$ is $7635.1 \mathrm{~nm}\left(\angle \mathrm{FO} d=127.252^{\circ}\right)$, the initial course angle $(C)$ is $573.9^{\circ} \mathrm{E}\left(\angle d^{\prime} \mathrm{O}^{\prime} \mathrm{T}_{\mathrm{H}}=73.943^{\circ}\right)$, and the initial course $\left(C_{n}\right)$ is $106.1^{\circ}$.

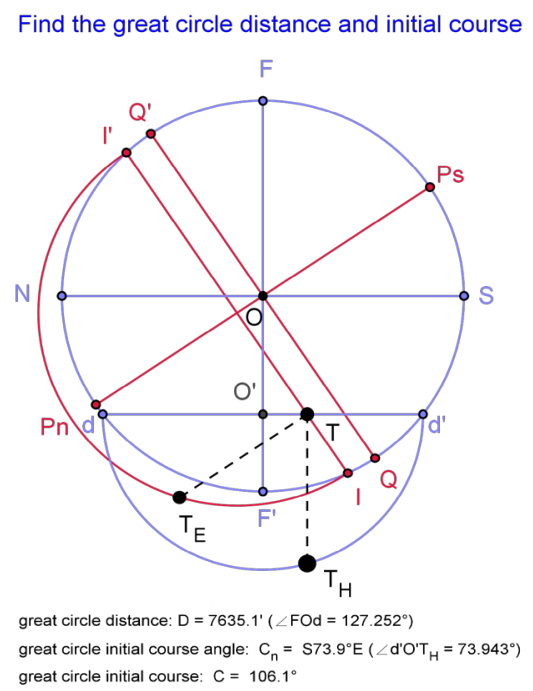

Fig. 13. Result of the graphical method to find the great circle distance and initial course in example

2) Find waypoints

- Condition 1: Find the latitude of waypoint $\left(L_{\mathrm{x}}\right)$ and the difference of longitude between the departure and waypoint $\left(D L o_{\mathrm{FX}}\right)$ when the great circle distance, which starts from the departure towards the waypoint $\left(D_{\mathrm{FX}}\right)$, is 2544.3 nautical miles ( $\mathrm{nm}$ ).

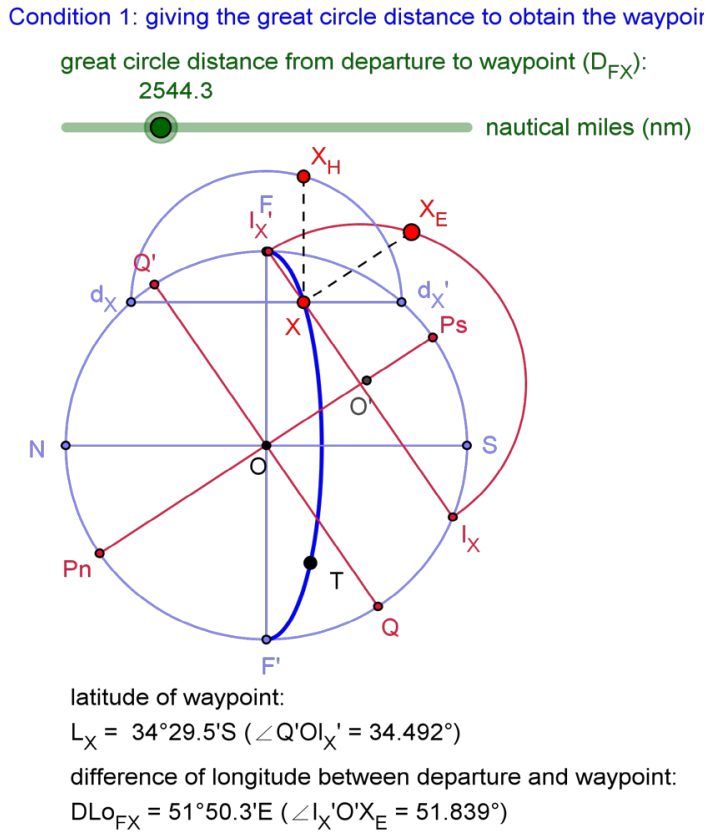

Fig. 14. Result of the graphical method to find the waypoint under condition 1 in example
As shown in Fig. 14, the results of calculation are that the latitude of waypoint $\left(L_{X}\right)$ is $34^{\circ} 29.5^{\prime} \mathrm{S}\left(\angle \mathrm{Q}^{\prime} \mathrm{O} l_{X}{ }^{\prime}=34.492^{\circ}\right)$, and the difference of longitude between the departure and waypoint $\left(D L o_{\mathrm{FX}}\right)$ is $51^{\circ} 50.3^{\prime} \mathrm{E}\left(\angle l_{X}{ }^{\prime} O^{\prime} X_{E}=51.839^{\circ}\right)$.

- Condition 2: Find the latitude of waypoint $\left(L_{x}\right)$ when the difference of longitude between the departure and waypoint $\left(D L o_{\mathrm{FX}}\right)$ is $78^{\circ} 47.0^{\prime} \mathrm{E}$.

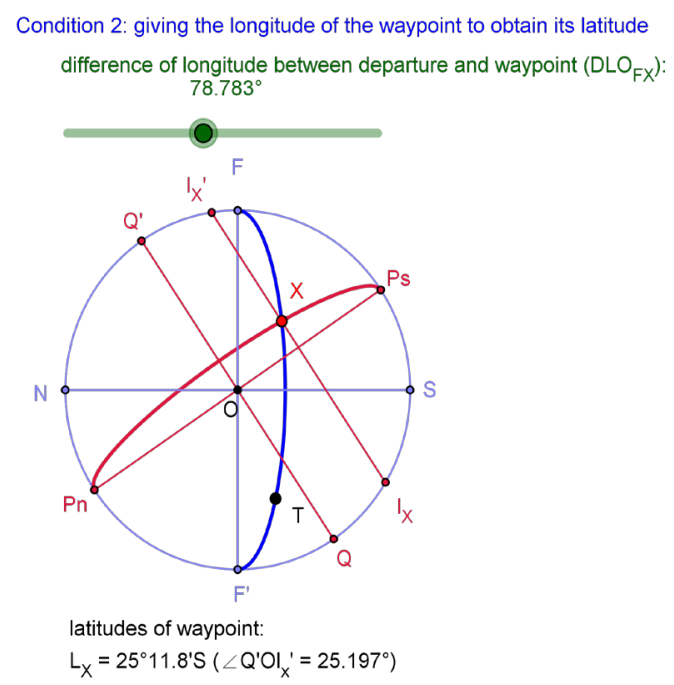

Fig. 15. Result of the graphical method to find the waypoint under condition 2 in example

As shown in Fig. 15, the result of calculation is that the latitude of waypoint $\left(L_{X}\right)$ is $25^{\circ} 11.8^{\prime} \mathrm{S}\left(\angle \mathrm{Q}^{\prime} \mathrm{O} l_{X}{ }^{\prime}=25.197^{\circ}\right)$.

- Condition 3: Find the difference of longitude between the departure and waypoint $\left(D L o_{\mathrm{FX}}\right)$ when the latitude of waypoint $\left(L_{\mathrm{x}}\right)$ is $13^{\circ} 30.1^{\prime} \mathrm{S}$.

As shown in Fig. 16, the result of calculation is that the difference of longitude from departure to waypoint $\left(D L o_{\mathrm{FX}}\right)$ is $98^{\circ} 46.9^{\prime} \mathrm{E}\left(\angle l_{X}{ }^{\prime} \mathrm{O}^{\prime} X_{\mathrm{E}}=98.782^{\circ}\right)$.

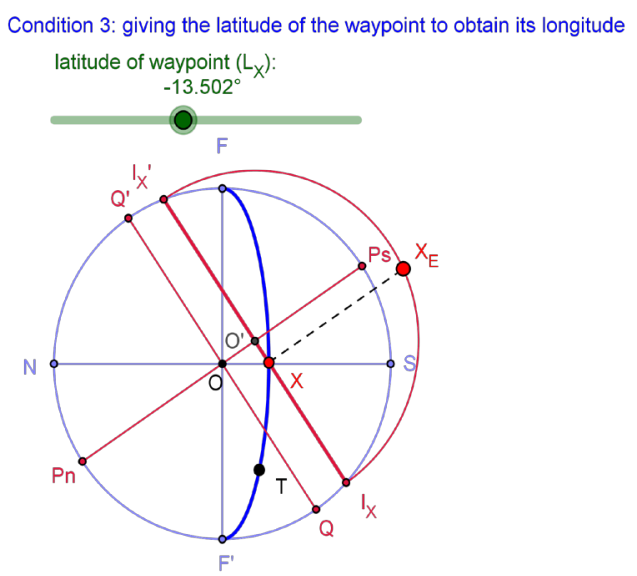

difference of longitude between departure and waypoint: $D L O_{F X}=98^{\circ} 46.9^{\prime} E\left(I_{X}^{\prime} O^{\prime} X_{E}=98.782^{\circ}\right)$

Fig. 16. Result of the graphical method to find the waypoint under condition 3 in example 
3) Find the vertex and the equator crossing point

As shown in Fig. 17, the results of calculation are that the latitude of vertex is $37^{\circ} 03.5^{\prime} \mathrm{S}\left(\angle \mathrm{Q}^{\prime} \mathrm{O} l_{V}{ }^{\prime}=37.059^{\circ}\right)$, and the difference of longitude between the departure and vertex $\left(D L o_{\mathrm{F} V}\right)$ is $27^{\circ} 19.3^{\prime} \mathrm{W}\left(\angle l_{V}{ }^{\prime} \mathrm{O}^{\prime} V_{\mathrm{E}}=27.322^{\circ}\right)$. Besides, the difference of longitude between the departure and equator crossing point $\left(D L o_{\mathrm{FE}}\right)$ is $117^{\circ} 19.3^{\prime} \mathrm{E}\left(\angle \mathrm{Q}^{\prime} \mathrm{O} E_{E}=117.322^{\circ}\right)$.

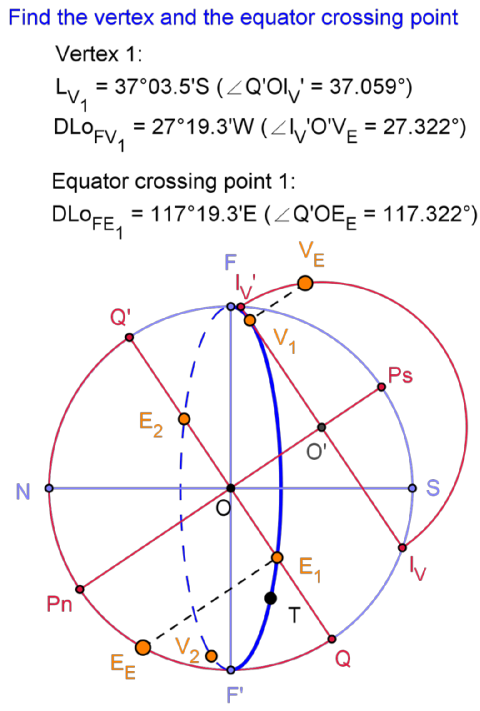

$$
\begin{aligned}
& \text { Vertex 2: } \\
& \mathrm{L}_{\mathrm{V}_{2}}=37^{\circ} 03.5^{\prime} \mathrm{N} \quad \mathrm{DLO} \mathrm{FV}_{2}=152^{\circ} 40.7^{\prime} \mathrm{E} \\
& \text { Equator crossing point } 2: \\
& \mathrm{DLO}_{\mathrm{FE}_{2}}=62^{\circ} 40.7^{\prime} \mathrm{W}
\end{aligned}
$$

Fig. 17. Result of the graphical method to find the vertex and the equator crossing point in example

In this example, we found that the results of calculation by using the computer graphing software to execute the graphical method (GM) are as accurate as by using the algebraic methods [4].

\section{ANALYSIS AND DISCUSSION}

The GM not only can accurately solve the GCR problems, but also can help us to analyze relative variables. When the latitude of departure $\left(L_{\mathrm{F}}\right)$, the latitude of destination $\left(L_{\mathrm{T}}\right)$, and the difference of longitude between departure and destination $(D L o)$ are known, these variables will determine the only GCR between the departure $(F)$ and destination $(T)$. In this paper, we assume that the $L_{\mathrm{F}}$ is $20^{\circ} \mathrm{N}$, the $L_{\mathrm{T}}$ is $32.5^{\circ} \mathrm{N}$, the $D L o$ is $63.3^{\circ} \mathrm{W}$, and try to change one variable value at a time. Through this example, we can analyze the relationship between these variables and the GCR.

First, when the latitude of departure $\left(L_{\mathrm{F}}\right)$ is changed, as shown in Fig. 18, the two axes of horizon coordinate will still be fixed, the two axes of earth coordinate (e.g., the equator) and the destination $(T)$ will rotate around the center $(O)$ on the diagram. When the latitude of departure $\left(L_{\mathrm{F}}\right)$ value decreases from $90^{\circ} \mathrm{N}$ to $90^{\circ} \mathrm{S}$ (e.g., $L_{\mathrm{F} 1}=90^{\circ} \mathrm{N}, L_{\mathrm{F} 2}=67.5^{\circ} \mathrm{N}$, $\left.\ldots, L_{\mathrm{F} 9}=90^{\circ} \mathrm{S}\right)$, the equator will rotate in a counterclockwise direction from $\overline{\mathrm{Q}_{1} \mathrm{Q}_{1}}$ to $\overline{\mathrm{Q}_{9} \mathrm{Q}_{9}}$, and the destination will rotate in a counterclockwise direction from $\mathrm{T}_{1}$ to $\mathrm{T}_{9}$. Thus, the latitude of departure $\left(L_{\mathrm{F}}\right)$ is the variable which links two coordinates.

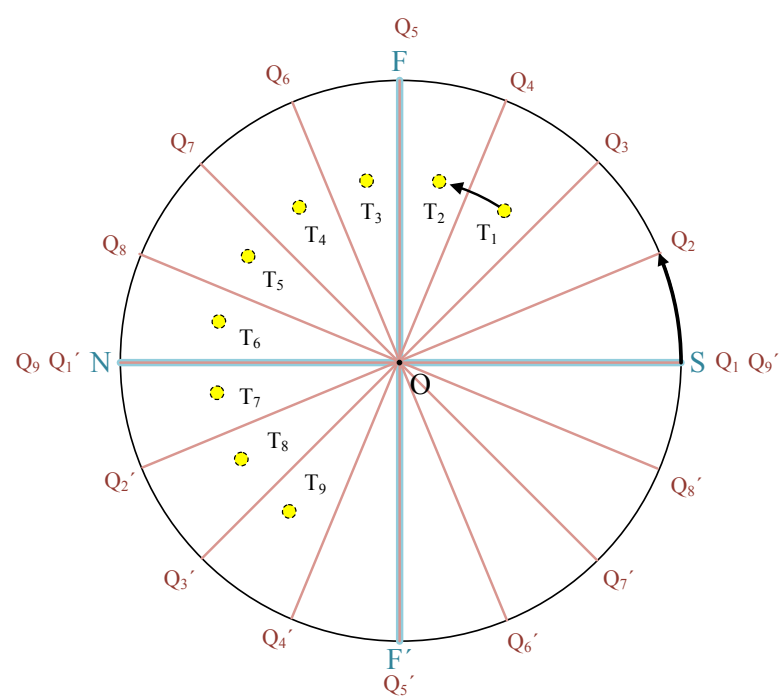

Fig. 18. Variable analysis for the latitude of departure

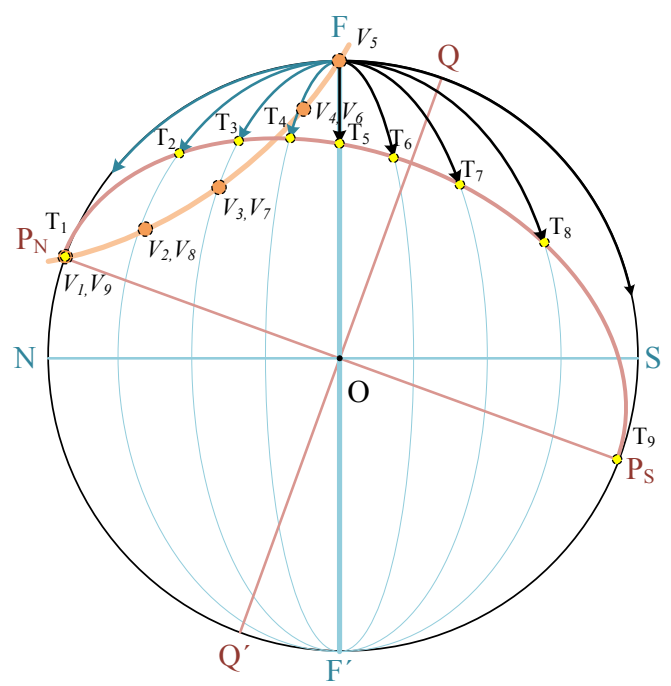

Fig. 19. Variable analysis for the latitude of destination

Next, when the latitude of destination $\left(L_{\mathrm{T}}\right)$ is changed, as shown in Fig. 19, the destination (T) will move along its meridian $\left(\widehat{\mathrm{P}_{\mathrm{N}} \mathrm{P}_{\mathrm{S}}}\right.$, the red curved line), and the vertex $(V)$ will move along a curve $\left(\widehat{\mathrm{P}_{\mathrm{N}} \mathrm{F}}\right.$, the orange curved line) between the departure $(\mathrm{F})$ and elevated pole (when the departure is in the north latitude, its elevated pole will be the north pole and vice versa). The move curve of the vertex validates the concept of the vertex circle that is proposed by Chiang and Tseng (1992) [6]. When the latitude of destination $\left(L_{\mathrm{T}}\right)$ value decreases from $90^{\circ} \mathrm{N}$ to $90^{\circ} \mathrm{S}$, the destination ( $\mathrm{T}$ ) will move along its meridian from $\mathrm{T}_{1}$ to $\mathrm{T}_{9}$, and the vertex $(\mathrm{V})$ will move along the curve between the departure (F) and the north pole $\left(\mathrm{P}_{\mathrm{N}}\right)$ from $\mathrm{V}_{1}$ to $\mathrm{V}_{9}$. The $\mathrm{V}_{1}$ to $\mathrm{V}_{4}$ are on the diagram, and the 
$\mathrm{V}_{5}$ to $\mathrm{V}_{9}$ are at the "back" side of the diagram. Furthermore, use the prime vertical $\left(\overline{\mathrm{FF}^{\prime}}\right)$ as a criterion. If the destination (T) and the elevated pole are on the same side, the voyage will sail along the GCR towards the vertex $(V)$; if the destination (T) and the elevated pole are on the opposite side, the voyage will sail along the GCR away the vertex $(V)$. Choosing the GCR which sails towards the vertex $(V)$ can obtain more apparent benefit. For example, the $\mathrm{T}_{1}$ to $\mathrm{T}_{4}$ and the north pole $\left(\mathrm{P}_{\mathrm{N}}\right)$ are on the left side of the prime vertical so that these four GCRs are towards their vertex $\left(V_{1}\right.$ to $\left.V_{4}\right)$; the $\mathrm{T}_{5}$ to $\mathrm{T}_{9}$ are on the right side of the prime vertical so that these five GCRs are away their vertex $\left(V_{5}\right.$ to $\left.V_{9}\right)$. Hence, the latitude of destination $\left(L_{\mathrm{T}}\right)$ is the major variable which decides the benefit of GCR.

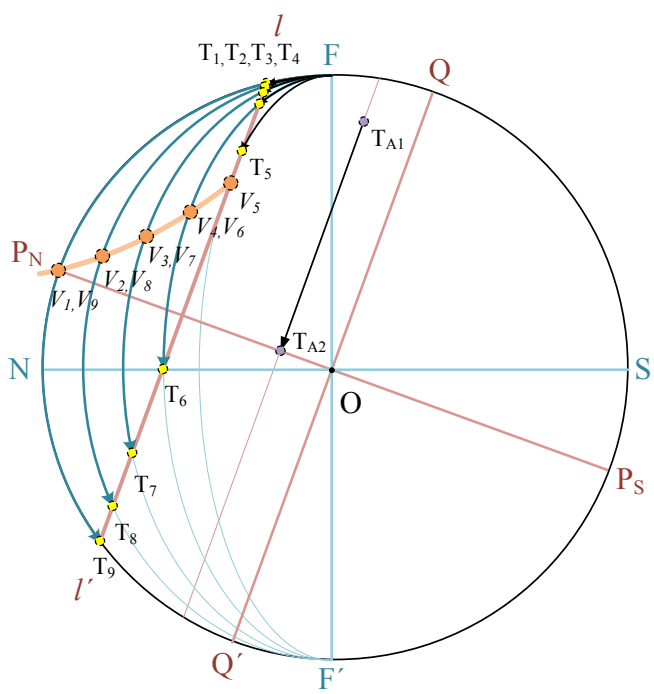

Fig. 20. Variable analysis for the difference of longitude between departure and destination

Finally, when the difference of longitude between the departure and destination (DLo) is changed, as shown in Fig. 20, the destination (T) will move along its POL $\left(\overline{l l^{\prime}}\right)$. Because of the limit of latitude $\left(L_{\mathrm{T}}\right)$, the vertex $(V)$ will move along a curve between the elevated pole and the POL of the destination. When the difference of longitude between the departure and destination ( $D L o$ ) value increases from $0^{\circ} \mathrm{W}$ to $180^{\circ} \mathrm{W}$, the destination $(T)$ will move along its POL $\left(\overline{l l^{\prime}}\right)$ from $\mathrm{T}_{1}$ to $\mathrm{T}_{9}$, and the vertex $(V)$ will move along the curve between the north pole $\left(\mathrm{P}_{\mathrm{N}}\right)$ and the POL of the destination $\left(\overline{l l^{\prime}}\right)$ from $\mathrm{V}_{1}$ to $\mathrm{V}_{9}$ If the difference of longitude ( $D L o$ ) value is smaller (e.g., $\mathrm{T}_{1}$ to $\mathrm{T}_{5}$ ), the voyage will not through the vertex (e.g., $\mathrm{V}_{1}$ to $\mathrm{V}_{5}$ ); if the difference of longitude value is larger (e.g., $\mathrm{T}_{1}$ to $\mathrm{T}_{9}$ ), the voyage will through the vertex (e.g., $\mathrm{V}_{6}$ to $\mathrm{V}_{9}$ ). Choosing the GCR which sails through the vertex $(V)$ can obtain more apparent benefit. In addition, if the latitude of destination $\left(L_{\mathrm{T}}\right)$ value is lower than the latitude of departure $\left(L_{\mathrm{F}}\right)$, increasing sufficient difference of longitude value will let the destination ${ }_{\mathrm{T}}$ ) on the same side of the elevated pole (e.g., $\mathrm{T}_{\mathrm{A} 1}$ moves to $\mathrm{T}_{\mathrm{A} 2}$ ). It will help the voyage to sail towards even through the vertex $(\mathrm{V})$. Therefore, the difference of longitude between the departure and destination $(D L o)$ is the auxiliary variable which decides the benefit of GCR.

\section{CONCLUSIONS}

In this paper, the graphical method (GM) which applies the concepts of the celestial meridian diagram (CMD) not only can help us to combine the two coordinate systems of the great circle route (GCR) but also can let the earth be projected onto a tangent plane. Because of the latitude $\left(L_{\mathrm{T}}\right)$ can be found by measuring the central angle of the projected meridian of departure (MOD), the difference of longitude $(D L o)$ can be obtained by measuring the central angle of the spherical parallel of latitude (POL), the great circle distance $(D)$ can be found by measuring the central angle of the projected principal vertical, and the great circle initial course $(C)$ can be obtained by measuring the central angle of the spherical distance circle, using the GM can obtain the all relative information of GCR. Applying the computer graphing software to execute the GM can obtain accurate results without using any algebraic equations. In addition, it can even analyze the relationship between these variables and the GCR. The three important rules are illustrated as follows: First, the latitude of departure $\left(L_{\mathrm{F}}\right)$ is the variable which links the earth coordinate and the horizon coordinate; the benefit of GCR is decided by the latitude of destination $\left(L_{\mathrm{T}}\right.$ and the difference of longitude between the departure and destination $(D L o)$. The former is the major variable and the latter is the auxiliary variable. Second, use the prime vertical as a criterion, when the destination (T) and the elevated pole are on the same side, the benefit of GCR is more apparent. When a GCR sails through the vertex $(V)$, its benefit is the most obvious. Third, if the latitude of destination $\left(L_{\mathrm{T}}\right)$ value is lower than the latitude of departure $\left(L_{\mathrm{F}}\right)$, increasing sufficient difference of longitude value will help the voyage to sail towards even through the vertex $(V)$. Accordingly, the GM can emphasize the rotational surface and help us to comprehend the GCR by using the diagram.

\section{BIBLIOGRAPHY}

1. Bowditch, N.: The American Practical Navigator, 2002 Bicentennial Edition, National Imagery and Mapping Agency, Bethesda, Maryland, 2002.

2. Chen, C. L.: A Systematic Approach for Solving the Great Circle Track Problems Based on Vector Algebra, Polish Maritime Research, 23(2), pp. 3-13, 2016.

3. Chen, C. L., Hsieh, T. H. and Hsu, T. P.: A novel approach to solve the great circle track based on rotation transformation, Journal of Marine Science and Technology, 23(1), pp. 13-20, 2015.

4. Chen, C. L., Hsu, T. P. and Chang, J. R.: A novel approach to great circle sailings: the great circle equation, The Journal of Navigation, 57(2), pp. 311-320, 2004.

5. Chen, C. L., Liu, P. F. and Gong, W. T.: A simple approach to great circle sailing: the COFI method, The Journal of Navigation, 67(3), pp. 403-418, 2014. 
6. Chiang, C. H. and Tseng, A. Y.: Some ideas on calculating great circle sailings, The Journal of Navigation, 45(1), pp. 136-138, 1992.

7. Cutler, T. J.: Dutton's Nautical Navigation, Fifteenth Edition, Naval Institute Press, Maryland, 2004.

8. Earle, M. A.: Vector solutions for great circle navigation, The Journal of Navigation, 58(3), pp. 451-457, 2005.

9. Miller, A. R., Moskowitz, I. S. and Simmen, J.: Traveling on the curved earth, NAVIGATION, Journal of the Institute of Navigation, 38(1), pp. 71-78, 1991.

10. Nastro, V. and Tancredi, U.: Great circle navigation with vectorial methods, The Journal of Navigation, 63(3), pp. 557-563, 2010.

11. Royal Navy: The Admiralty Manual of Navigation: The Principles of Navigation, Volume 1, Tenth Edition, Nautical Institute, London, 2008.

12. Sa, S. H.: Navigation, Volume 2, Wensheng Book Store, Taiwan, 2010. (In Chinese)

13. Tseng, W. K. and Chang, W. J.: Analogues between 2D linear equations and great circle sailing, The Journal of Navigation, 67(1), pp. 101-112, 2014.

14. UNCTAD: Review of Maritime Transport, Geneva: United Nations, 2015.

\section{NOMENCLATURE}

$\mathrm{P}$

Z

$S$

Dec

$T$

$H$

$H_{\mathrm{P}}$

$A z$

$\mathrm{P}_{\mathrm{N}}$

F

$\mathrm{T}$

$\mathrm{T}_{\mathrm{E}}$

$\mathrm{T}_{\mathrm{H}}$

$X$

$X_{\mathrm{E}}$

$X_{\mathrm{H}}$

V

$V_{\mathrm{E}}$

E

$E_{\mathrm{E}}$

$L_{\mathrm{F}}$

$L_{\mathrm{T}}$
$L_{\mathrm{X}} \quad$ latitude of waypoint

$L_{\mathrm{V}} \quad$ latitude of vertex

DLo difference of longitude between departure and destination

$D L o_{\mathrm{FX}} \quad$ difference of longitude between departure and waypoint

$D L o_{\mathrm{FV}} \quad$ difference of longitude between departure and vertex

$\mathrm{DLO}_{\mathrm{FE}}$ difference of longitude between departure and equator crossing point

D great circle distance from departure to destination

$D_{\mathrm{FX}} \quad$ great circle distance from departure to waypoint

$C \quad$ great circle initial course angle

$C_{n} \quad$ great circle initial course

$O \quad$ center of the diagram

$\mathrm{O}^{\prime} \quad$ center of a semicircle

$\overline{\mathrm{P}_{\mathrm{N}} \mathrm{P}_{\mathrm{S}}} \quad$ polar axis

$\overline{\mathrm{QQ}^{\prime}} \quad$ equator

$\overline{l l}^{\prime}, \widehat{l l}^{\prime}$, parallel of latitude

$\overline{l_{X} l_{X}}, l_{X} l_{X}{ }^{\prime} \quad$ parallel of latitude of the waypoint

$\frac{l_{X} l_{X}}{l_{V} l_{V}} \quad$ parallel of latitude of the vertex

$M_{1}, M_{2}, M_{3}$ points on a meridian

$\overline{\mathrm{FF}^{\prime}} \quad$ prime vertical

$\overline{\mathrm{NS}}$ horizon

$\overline{d d^{\prime}}, \widehat{d d} \quad$ distance circle

$\overline{d_{X} d_{X}}, \widehat{d_{X} d_{X}}$, distance circle of a waypoint

$G_{1}, G_{2}, G_{3}$ points on a vertical circle

\section{CONTACT WITH THE AUTHOR}

\author{
Tsung-Hsuan Hsieh \\ e-mail:d98521003@ntu.edu.tw \\ Department of Civil Engineering \\ National Taiwan University
}

No. 1, Sec. 4, Roosevelt Road, Taipei, 10617

TAIWAN 